\title{
Influence of Stack Orientations on the Static Strengths of SPR Joints
}

\author{
Dezhi Li ${ }^{1, *}$, Geraint Williams ${ }^{1}$, Li Han² and Mike Shergold ${ }^{2}$ \\ ${ }^{1}$ WMG, University of Warwick, Coventry, CV4 7AL, UK \\ 2Jaguar Engineering Centre, Jaguar Land Rover, Coventry, CV3 4LF, UK \\ * Corresponding author, e-mail: dezhi.li@warwick.ac.uk
}

Keywords:self-piercing riveting (SPR), stack orientation, lap shear, T peel

\begin{abstract}
To improve fuel efficiency and reduce greenhouse gas emissions, lightweight materials, such as aluminium alloys, are used increasingly in automotive manufacturing. Currently, self-piercing riveting (SPR) is a major technology used by manufacturers to join aluminium body structures due to its advantages over other joining technologies. When different materials are joined together, different stack orientations can be used. To find out the influence of stack orientations on joint strength for further structure optimization, in this paper the joint quality and the static strengths of three pairs of twothickness stacks with different orientations were studied. The results showed that by joining the thicker material as a bottom material rather than a top material, joint interlocks were improved. As to the mechanical strength, for the stacks with both top and bottom sheets as AA5754, when a thicker sheet was joined as a bottom material, the joints had stronger static strength, and for stacks with $3.0 \mathrm{~mm}$ AA5754 and $2.0 \mathrm{~mm}$ AA6008T61, when the $3.0 \mathrm{~mm}$ AA5754 was joined as a bottom material, the joints had stronger static lap shear strength but weaker static $\mathrm{T}$ peel strength.
\end{abstract}

\section{Introduction}

To improve fuel efficiency and reduce greenhouse gas emissions, reducing vehicle weight continues to be a key objective in the automotive industry. A $10 \%$ reduction in vehicle weight offers fuel savings of $5-7 \%$ (in mpg), provided the vehicle's powertrain is also downsized. As a result, lightweight materials, such as aluminium and magnesium alloys, are used increasingly for automotive body structures. Currently, self-piercing riveting (SPR) is one of the major technologies used by the automotive industry to join aluminium body structures. Research in this area has shown that self-piercing riveting of aluminium alloys gives joints of comparable static strength and superior fatigue performance to spotwelding [1-3].

There have been a few reported studies on joining high strength steel with aluminium alloys using SPR. Abe et al. [4, 5] studied the feasibility of joining aluminium alloy with mild steel or high strength steel. Their results showed that for a combination of steel and aluminum, SPR is more capable of joining aluminum alloy as the bottom layer. Mori et al. [6] investigated SPR of ultra-high strength steel with aluminium alloy. They suggested that by optimizing the profile of die, ultra-high strength steel as either top or bottom sheet could be successfully joined, and problems such as button fracture and lack of interlock could be resolved. Han et al. [7] studied the quality and mechanical properties of SPR joints containing aluminium alloy and high strength low alloy steel. So far, there is a limited literature in public domain regarding the influence of stack orientation on the mechanical strength of SPR joints. Han et al. [8] studied the influence of stack orientation on the strength of resistance spot welded aluminium joints, and they found that the influence was negligible. However, for SPR this influence will be different due to different joint structures and joint forming mechanisms. In this paper, the influence of 
stack orientation on the joint quality and the static strengths of three pairs of two-thickness stacks were studied.

\section{Experimental Procedures}

\section{Materials}

$2.0 \mathrm{~mm}, 2.5 \mathrm{~mm}$ and $3.0 \mathrm{~mm}$ thick AA5754, $2.0 \mathrm{~mm}$ thick AA6008 T61 were used in this study. AA5754 has a standard PT2 AL070 surface condition (Electrically cleaned after rolled to final gauge, and then pretreated with PT2 process and applied with AL070 dry lubricant); whilst AA6008T61 has mill finished surface. The compositions of AA5754 and AA6008T61 are listed in Table 1. AA5754 and AA6008T61 have the yield strength, ultimate tensile strength and elongation as $109 \mathrm{MPa}, 240 \mathrm{MPa}$, $24 \%$ and $215 \mathrm{MPa}, 296 \mathrm{MPa}, 24 \%$, respectively. AA6008T61 has a much higher strength than AA5754.

\section{Sample Preparation}

For all stacks, steel rivets with a countersunk head and mechanical zinc/tin surface coating were used. The rivets were supplied by Henrob Ltd, and all samples were produced using Henrob Ltd servo-driven riveting equipment. The stacks were expressed as 'top-sheet + bottom-sheet', for example, the 3.0AA5754 + 2.5AA6008T61 stack meant $3.0 \mathrm{~mm}$ AA5754 was used as the top material and $2.5 \mathrm{~mm}$ AA6008T61 was used as the bottom material. For all joints, a flat bottom die (designated as DF) with cavity diameter of $9 \mathrm{~mm}$ and depth of $2 \mathrm{~mm}$ was used. Different rivets were used to comply with different stack total thickness and material strength and various riveting velocities were applied to achieve proper rivet head heights. For a specific pair of stacks with different orientations, same rivet and setting velocity were used.

Table 1 Nominal compositions of AA6008 and AA5754 (balance Al).

\begin{tabular}{|l|l|l|l|l|l|l|l|l|l|l|}
\hline & $\mathrm{Mg}$ & $\mathrm{Mn}$ & $\mathrm{Cu}$ & $\mathrm{Fe}$ & $\mathrm{Si}$ & $\mathrm{Ti}$ & $\mathrm{Cr}$ & $\mathrm{Zn}$ & $\mathrm{V}$ & Others \\
\hline AA6008 & $0.4-0.7$ & $\begin{array}{l}0- \\
0.3\end{array}$ & $\begin{array}{l}0- \\
0.3\end{array}$ & $\begin{array}{l}0- \\
0.35\end{array}$ & $\begin{array}{l}0.5- \\
0.9\end{array}$ & $0-0.1$ & $0-0.3$ & $0-0.2$ & $\begin{array}{l}0.05- \\
0.2\end{array}$ & $0-0.15$ \\
\hline AA5754 & $2.6-3.6$ & $\begin{array}{l}0- \\
0.5\end{array}$ & $\begin{array}{l}0- \\
0.1\end{array}$ & $0-0.4$ & $0-0.4$ & $0-0.15$ & $0-0.3$ & $0-0.2$ & - & $0-0.15$ \\
\hline
\end{tabular}

All samples were made using custom designed fixtures to reduce any variations of rivet position. Specimen geometry and dimensions for static lap shear and T-peel tests are shown in Fig. 1. During the preparation of specimens, coupons were cut from sheet such that the longitudinal direction of coupons coincides with the rolling direction of sheet metal. Double joints SPR samples were then fabricated using the custom-designed fixtures.

\section{Mechanical Strength Tests}

Static lap shear and T-peel tests were performed using a standard Instron tensile test machine at a 10 $\mathrm{mm} / \mathrm{min}$ cross-head speed. In order to minimize coupon bending and to ensure alignment of the load paths, spacers of various thicknesses were used at both ends of the lap-shear samples. At least 3 samples were tested at each condition.

\section{Joint Quality Analysis}

Joint quality of specimen with different stacks was inspected through cross-sections. A special fixture was used to ensure all joints were vertically cross-sectioned across the center of the rivets. Following 
sectioning, the joint features were measured and analyzed with respect to rivet head height, interlock and minimum remaining material thickness using an optical microscope and a4i image analysis software, a dedicated image capture, archive, analysis and data basing package supplied by Aquinto.

\section{Results}

\section{Joint Quality}

The acceptable joint quality criteria: rivet head height: -0.5 to $0.3 \mathrm{~mm}$, average interlock distance: $\geq 0.4$ $\mathrm{mm}$, and minimum remaining bottom material thickness: $\geq 0.2 \mathrm{~mm}$, were used to judge joint quality. Figure 2 shows the cross-sections of two thickness stacks containing a layer of AA5754 and a layer of AA6008T61. Fig. 2a shows that for the 3.0AA5754+2.0AA6008T61 stack the average interlock distance of $0.31 \mathrm{~mm}$ is not sufficient. Fig. $2 \mathrm{~b}$ shows that the joint quality of the 2.0AA6008T61+3.0AA5754 stack is very good.

Fig. 3 shows the cross-sections of two thickness stacks containing different thickness of AA5754. Fig. 3 a shows that for the $(3+2)$ AA5754 stack the average interlock distance of the joints, $0.31 \mathrm{~mm}$, was not sufficient. On the contrary, for the (2+3)AA5754 stack, the average interlock distance, $0.67 \mathrm{~mm}$ as shown in Fig. $3 \mathrm{~b}$, was very large. When the thickness difference of the top and bottom sheets reduced from $1 \mathrm{~mm}$ to $0.5 \mathrm{~mm}$, the joint quality for the (3+2.5)AA5754 and (2.5+3)AA5754 stacks was similar with the average interlock distances as $0.45 \mathrm{~mm}$ and $0.47 \mathrm{~mm}$ as shown in Fig. 3c and d, respectively, although the interlock distance for the stack with thinner sheet on top was still slightly larger.

\section{Mechanical Strength}

From Table 2, it can be seen that for stacks in which both top and bottom sheets were AA5754 (stacks 1 to 4) when a thicker sheet was joined as a bottom material the joints would have higher lap shear and $\mathrm{T}$ peel strengths. For $3.0 \mathrm{~mm}$ AA5754 and $2.0 \mathrm{~mm}$ AA6008T61 stacks, the joints with $2.0 \mathrm{~mm}$ AA6008T61 joined as a bottom material had better T peel strength but poorer lap shear strength than those with $2.0 \mathrm{~mm}$ AA6008T61 joined as a top material. It can also be seen that when a stronger material AA6008 was used to replace AA5754, both the lap shear and T peel strength were increased.
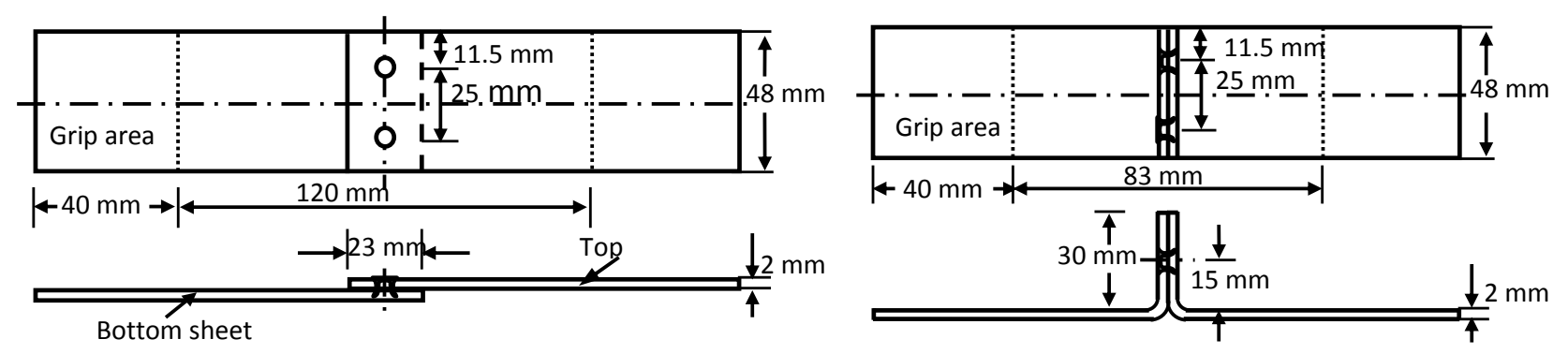

Figure 1 Specimen geometry for static lap shear and T peel tests. 

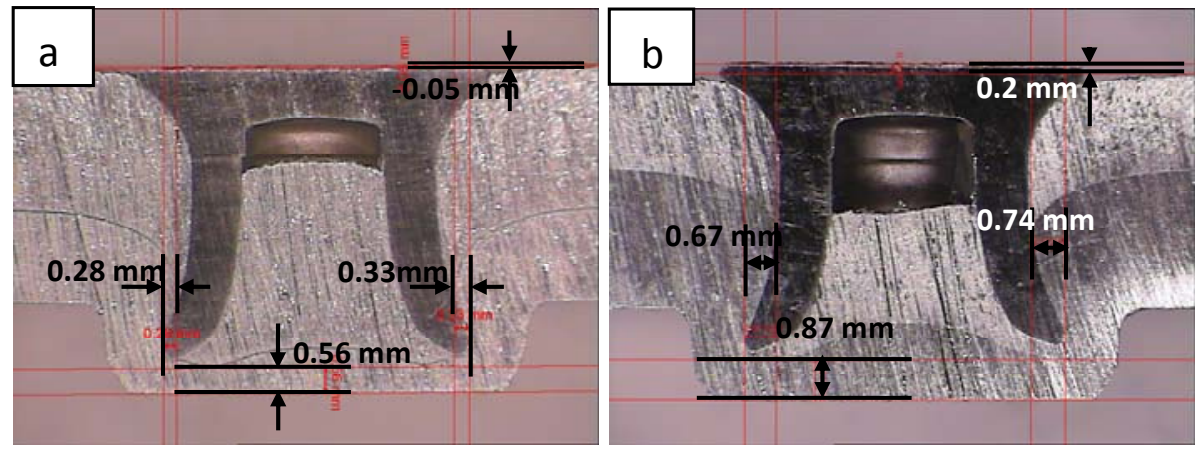

Figure 2 Cross-sections of SPR joints from different AA5754 and AA6008T61 stacks, a) 3.0AA5754+ 2.0AA6008T61 and b) 2.0AA6008T61 +3.0AA5754.
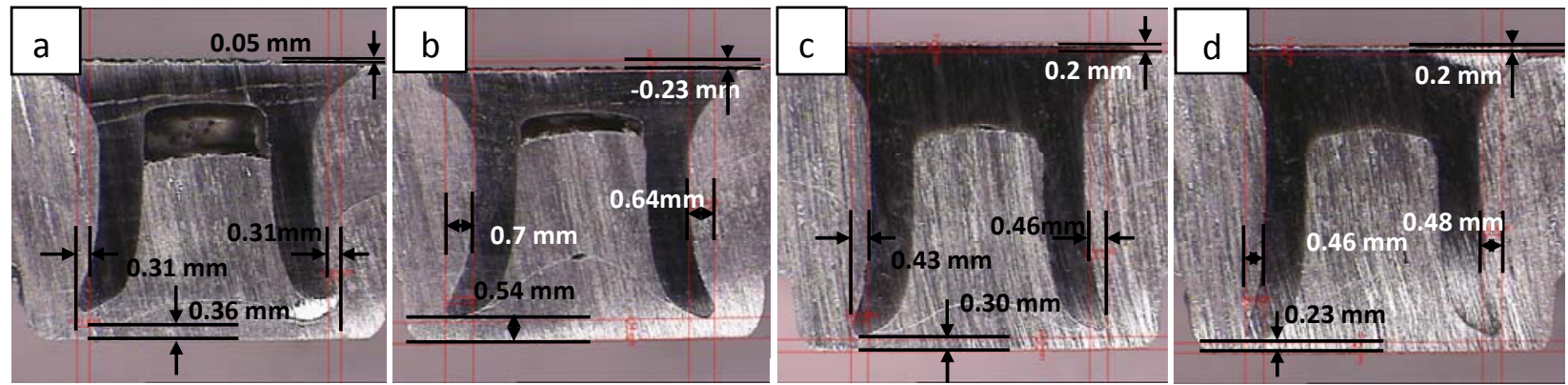

Figure 3 Cross-sections of SPR joints from different AA5754 stacks, a) $(3.0+2)$ AA5754, b) (2.0+3) AA5754, c) $(3+2.5)$ AA5754, and d) $(2.5+3)$ AA5754.

Table 2 Static lap shear strengths of joints from different stacks.

\begin{tabular}{|l|l|l|l|}
\hline Stack ID & Material and orientation & Lap shear strength, KN & T peel strength, KN \\
\hline 1 & $(3.0+2.5)$ AA5754 & $10.5 \pm 0.29$ & $3.72 \pm 0.14$ \\
\hline 2 & $(2.5+3.0)$ AA5754 & $10.78 \pm 0.13$ & $5.06 \pm 0.29$ \\
\hline 3 & $(3.0+2.0)$ AA5754 & $8.88 \pm 0.09$ & $2.32 \pm 0.11$ \\
\hline 4 & $(2.0+3.0)$ AA5754 & $9.63 \pm 0.21$ & $3.07 \pm 0.18$ \\
\hline 5 & 3.0AA5754+2.0AA6008 & $10.02 \pm 0.2$ & $3.4 \pm 0.12$ \\
\hline 6 & 2.0AA6008+3.0AA5754 & $10.90 \pm 0.21$ & $3.18 \pm 0.13$ \\
\hline
\end{tabular}

\section{Discussion}

SPR joints are complex structures including various material interfaces and interlocks. Several factors can influence the joint quality and joint strength, including stack orientations, sheet material strength and thickness, stack thickness, rivet length and hardness, die profiles, frictions between the sheet material interfaces, frictions between rivet and sheet materials and rivet inserting forces etc. Among these, the stack orientation is an important one, especially for stack rivetability. 


\section{Influence of Stack Orientation on Joint Quality}

From Figs. 2 and 3, it can be seen that for selected rivet and die combination when a thinner material was joined as the bottom material, it was possible that the joints would have insufficient interlock distance, and by reserving the stack orientation the interlock distance for the joints were greatly increased. It should be pointed out that by selecting a different rivet and die combination the joint quality can be improved when a thinner sheet was used as a bottom material, but here we can see that stacks with thicker materials as a bottom material can be self-piercing riveted more easily or have larger process windows. For the (2.5+3)AA5754 and $(3+2.5)$ AA5754 stacks, because the thickness difference between the two materials was smaller, although there was still some interlock distance increase by putting the thicker material at the bottom, this influence was much less.

It can be seen that for the pair of stacks with different orientations, the overall flare of rivet legs was similar, but when a thinner material was joined as the bottom material, the flare of rivet into the bottom material (interlock) was much smaller. It can be seen that the penetration of rivet into the bottom material was shallower for the joints with a thinner material joined as the bottom material, and this penetration distance was the main factor that influenced the interlock distance.

The deformation of rivet legs was also influenced by other factors, such as material thickness and strength, rivet length and hardness, and die profiles etc. In this study, for the pair of stacks with reversed orientation, the same rivet and die were used, so rivets and die were not the influence factors. When a top material is thicker or stronger, the pierced part from the top sheet will not be easily pushed inside the rivet center cavity, leaving a big gap between the top of the pierced part and the rivet head, as shown in Figs. $2 \mathrm{~b}$ and $3 \mathrm{a}$. This will blunt the forwarding part of rivet (with the pierced material stuck at the entrance of the rivet cavity) and make the penetration of rivet into the bottom sheet harder. Subsequently, higher forces will be required to set the rivet into the stack with the same distance. However, these factors are not believed to be the main factors in this study.

\section{Influence of Stack Orientation on Quasi-static Joint Strength}

Fig. 4 shows the facture interfaces of the (2.5+3)AA5754 and (3+2.5)AA5754 stacks after static lap shear and $\mathrm{T}$ peel tests. It can be seen that for the $(2.5+3) \mathrm{AA} 5754$ stack, the specimens failed by rivets being pulled out from bottom sheets with top sheet deformed in lap shear tests and failed by rivets being pulled out from top sheets in T peel tests. For the $(3+2.5)$ AA5754 stack, in both lap shear and T peel tests the specimens failed by rivets being pulled out from bottom sheets. All lap shear specimens failed by rivet being pulled out from the bottom sheet, which is controlled by interlock distance. Because the $(2.5+3)$ AA5754 stack had slightly better interlock than the $(3+2.5)$ AA5754 stack, its lap shear strength was slightly better. As to these two stacks, the T peel strength was controlled by both the interlocks from the bottom sheet (rivet flare) and the top sheet (rivet head). All joints failed on the thinner sheet side. The (2.5+3)AA5754 stack had better T peel strength than the $(3+2.5)$ AA5754 stack, because the force required to pull out the rivet from the top sheet of the $(2.5+3)$ AA5754 stack was higher than that required to pull out the rivet from the bottom sheet of the $(3+2.5) \mathrm{AA} 5754$ stack in T peel configure.

Fig. 5 shows that the facture interfaces of the (2+3)AA5754 and (3+2)AA5754 stacks after static lap shear and T peel tests. It can be seen that for the (2+3)AA5754 stack, the specimens failed by rivets being pulled out from the top sheet or by tearing of the top sheet through the punched holes in lap shear tests, and failed by rivets being pulled out from the top sheet in T peel tests. For the (3+2)AA5754 stack, in both lap shear and $\mathrm{T}$ peel tests the specimens failed by rivets being pulled out from the bottom sheet. The failure of SPR joints during static tests is the competition of interlocking strength between the rivet head/top sheet interface and the rivet legs/bottom sheet interface. For these two stacks, all specimens failed at the thinner sheet, which indicated that the interlocking strength on the thinner sheet side was 
weaker. It is believed in this circumstance, between the two weaker interlocks, the interlocking strength between the top sheet and the rivet head in the joints for the (2+3)AA5754 stack was stronger than the that between the rivet legs and the bottom sheet in the joints for the $(3+2) A A 5754$ stack. As a results, the (2+3)AA5754 stack had better lap shear and T peel strength than the (3+2)AA5754 stack.
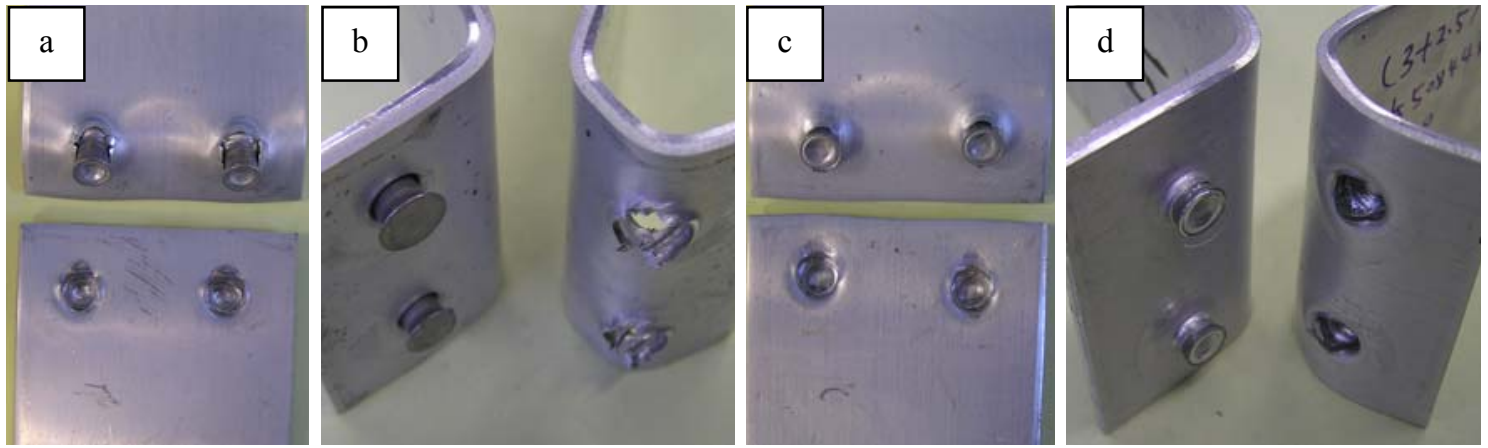

Figure 4 Fracture interfaces of $2.5 \mathrm{~mm}$ and $3.0 \mathrm{~mm}$ AA5754 stacks after static tests, a) (2.5+3)AA5754lap shear, b) (2.5+3)AA5754-T peel, c) (3+2.5)AA5754-lap shear, and d) (3+2.5)AA5754-T peel.
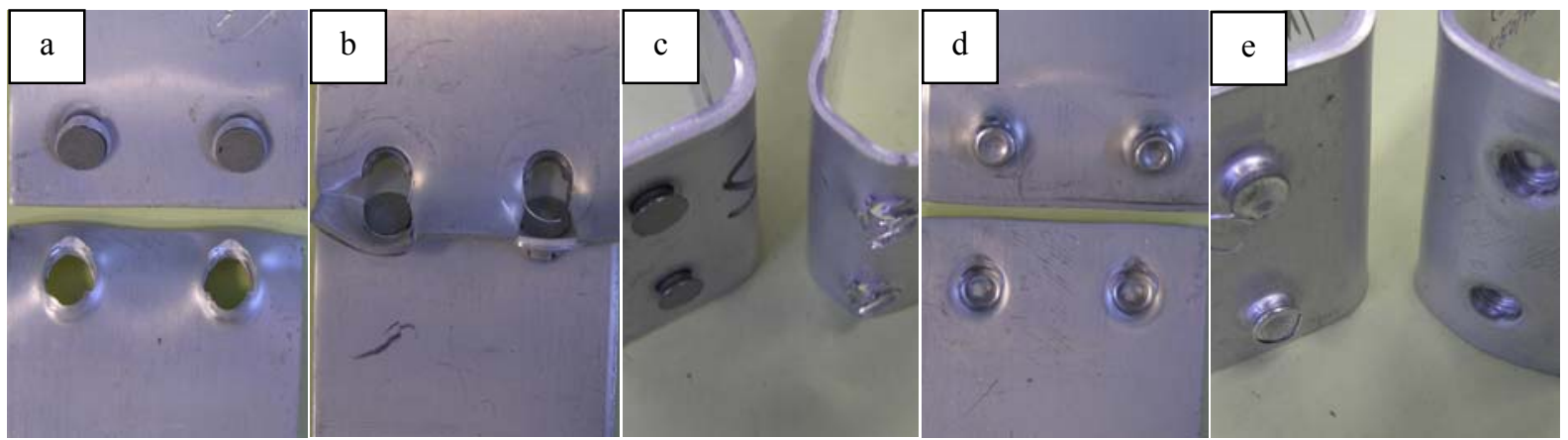

Figure 5 Fracture interfaces of $2.0 \mathrm{~mm}$ and $3.0 \mathrm{~mm}$ AA5754 stacks after static tests, a) b) (2+3)AA5754lap shear, c) (2+3)AA5754-T peel, d) (3+2)AA5754-lap shear, and e) (3+2)AA5754-T peel.
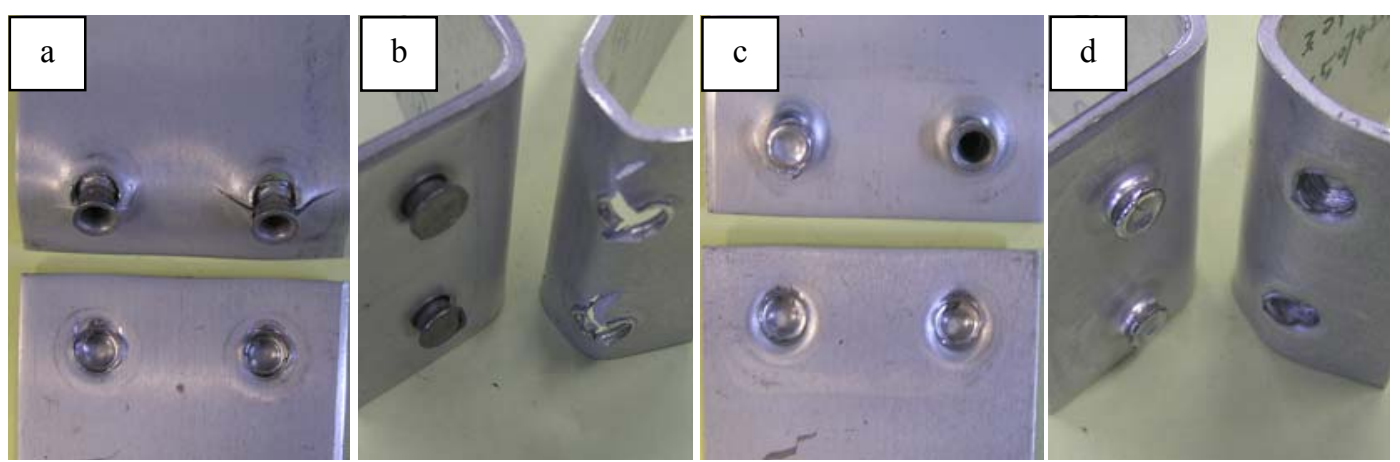

Figure 6 Fracture interfaces of $2.0 \mathrm{~mm}$ AA6008T61 and $3.0 \mathrm{~mm}$ AA5754 stacks after static tests, a) 2AA6008T61+3AA5754-lap shear, b) 2AA6008T61+3AA5754-T peel, c) 3AA5754+2AA6008T61-lap shear, and d) 3AA5754+2AA6008T61-T peel.

For the stacks with the top and the bottom materials with the same material, similar results were reported by Porcaro et al. [9], and they found that the use of a thicker material (AA6060) as the bottom 
sheet would normally produce the joints with higher strength than the use of a thinner material (AA6060) as the bottom sheet.

Fig. 6 shows that the facture interfaces of the 2AA6008T61+3AA5754 and 3AA5754+2AA6008T61 stacks after static lap shear and T peel tests. It can be seen that for the 2AA6008T61+3AA5754 stack, the specimens failed by rivets being pulled out from the bottom sheet with the top sheet cracked in lap shear tests and failed by rivets being pulled out from the top sheet with large cracks in it in $T$ peel tests. For the 3AA5754+2AA6008T61 stack, in both lap shear and T peel tests the specimens failed by rivets being pulled out from the bottom sheets. Although the AA6008 and AA5754 had the same elongation $(24 \%)$ based on dog-bone tensile tests, it was noticed that, due to its chemical compositions and microstructures, AA6008 had more tendency to crack [10]. It is believed that this cracking issue made the T peel strength of the 2AA6008T61+3AA5754 stack weaker that of the reversed stack. In both stacks, all specimens failed by rivet being pulled out from the bottom sheet in lap shear tests, which meant that the lap shear strength was controlled by the interlock distances. Since the interlock for the 2AA6008T61+3AA5754 stack was much larger than that for the 3AA5754+2AA6008T61 stack, the lap shear strength of the former was larger.

Generally speaking, stack orientation can influence SPR joint strength, but other factors can influence joint strength as well, and this influence will be different for different stacks with different SPR process parameters. The failure mode and joint strength of a SPR joint is decided by the interlocking strength of the rivet head, the interlocking strength of the flared rivet legs, rivet strength and the bearing strength of the substrates. Ultimately, they are decided by the joint features, including rivet head height, interlock distance and minimum bottom material thickness, and the material thickness and strength.

\section{Conclusions}

The influence of stack orientation on the joint quality and static strength was studied, and the following conclusions can be drawn:

1. Stacks with a thicker material as the bottom sheet have better rivetability/larger process windows than stacks with a thinner material as the bottom sheet.

2. For the all AA5754 stacks studied, the stacks with a thicker material as the bottom sheet had better lap shear and T peel strength.

3. For the mixed AA6008T61 and AA5754 stacks studied, the stacks with $3 \mathrm{~mm}$ AA5754 as a bottom material had better lap shear but poorer T peel strength, and the higher strength AA6008 substrate increased the joint strength, compared to the $2 \mathrm{~mm}$ and $3 \mathrm{~mm}$ AA5754 stacks.

\section{Acknowledgment}

The authors would like to thanks Innovate UK, the European Regional Development Fund and the Advanced West Midlands Fund, UK, for the support of this research. The authors would also like to thanks Henrob Ltd for providing the rivets for this research.

\section{References}

[1] Sun, X., E.V. Stephens, and M.A. Khaleel, Fatigue behaviors of self-piercing rivets joining similar and dissimilar sheet metals. International Journal of Fatigue, 2007. 29(2): p. 370-386.

[2] Booth, G.S., et al., Self-piercing riveted joints and resistance spot welded joints in steel and aluminium in SAE Technical Paper. 2000/01. p. 2681. 
[3] Krause, A.R. and A.R.A. Chernenkoff, A comparative study of the fatigue behaviour of spot welded and mechanically fastened aluminium joints, in SAE Technical Paper. 1995. p. 950710.

[4] Abe, Y., T. Kato, and K. Mori, Joinability of aluminium alloy and mild steel sheets by self piercing rivet. Journal of Materials Processing Technology, 2006. 177(1-3): p. 417-421.

[5] Abe, Y., T. Kato, and K. Mori, Self-piercing riveting of high tensile strength steel and aluminium alloy sheets using conventional rivet and die. Journal of Materials Processing Technology, 2009. 209(8): p. 3914-3922.

[6] Mori, K., et al., Plastic Joining of Ultra High Strength Steel and Aluminium Alloy Sheets by Self Piercing Rivet. CIRP Annals - Manufacturing Technology, 2006. 55(1): p. 283-286.

[7] Han, L. and A. Chrysanthou, Evaluation of quality and behaviour of self-piercing riveted aluminium to high strength low alloy sheets with different surface coatings. Materials \& Design, 2008. 29(2): p. 458-468.

[8] Han, L., et al., Effect of governing metal thickness and stack orientation on weld quality and mechanical behaviour of resistance spot welding of AA5754 aluminium. Materials \& Design, 2011. 32(4): p. 2107-2114.

[9] Porcaro, R., et al., The behaviour of a self-piercing riveted connection under quasi-static loading conditions. International Journal of Solids and Structures, 2006. 43(17): p. 5110-5131.

[10] Li, D., et al., Influence of Die Profiles and Cracks on Joint Buttons on the Joint Quality and Mechanical Strengths of High Strength Aluminium Alloy Joint. Advanced Materials Research, 2012. 548: p. 398-405. 